\title{
Thrombotic thrombocytopenic purpura with cardiac involvement after an allergic reaction to turmeric and cumin: a case report
}

\begin{abstract}
Background: Cuminum cyminum (Cumin) and Curcuma longa (Turmeric) are plant derived spices which are commonly used as a part of everyday recipes in variety of cuisines. They are known causes of platelet dysfunction but Thrombotic thrombocytopenic purpura (TTP) following their consumption has never been reported in the literature. Primary cardiac involvement in TTP is a rare but fatal complication and may present as AMI, heart failure or arrhythmias.

Case Report: We report a case of a 46-year-old male who presented with features of spontaneous bleeding following consumption of Cumin and Turmeric. Laboratory investigations revealed hemolytic anemia, marked thrombocytopenia and blood smear showed schistocytes. In addition, cardiac involvement was noted as per changes seen on the EKG and markedly elevated Troponin I level. At this stage TTP was diagnosed which was subsequently confirmed by reduced ADAMTS13 activity level of $<10 \%$. He was successfully treated with Plasma Exchange (PEX) and Rituximab. PEX was complicated by 5 episodes of non-sustained Ventricular Tachycardia which was managed by Amiodarone.

Conclusion: Although the link between ADAMTS13 deficiency and idiopathic TTP has been well-established, the role of Cumin and Turmeric in the pathogenesis of TTP is not yet well clarified. We speculate that the acquired ADAMTS13 deficiency might have been triggered by those Spices. Myocardial injury is underdiagnosed in patients with TTP and is associated with higher mortality. Hence, patients diagnosed with TTP should be monitored for adverse cardiac events due to the high incidence of new AMI, arrhythmias and congestive heart failure.
\end{abstract}

Keywords: thrombotic thrombocytopenic purpura; myocardial damage by ttp; turmeric and cumin
Volume 7 Issue 3 - 2016

\section{Tun W Naing,' Ebad Ur Rahman,' Omar Abdulfattah Abdulfattah, ${ }^{2}$ Frances Schmidt ${ }^{2}$ 'Internal Medicine, Interfaith Medical Center, USA \\ 2Pulmonary and Critical care, Interfaith Medical Center, USA}

Correspondence: Tun W Naing, Internal Medicine, Interfaith Medical Center, 1545 Atlantic Avenue, Brooklyn, NY I 2 I 3 , USA, Tel 3479672304, Email victortwn2014@gmail.com

Received: November 18, 2016 | Published: November 28, 2016

\section{Introduction}

Cumin (Cuminum cyminum) and Turmeric (Curcuma longa) inhibit platelet aggregation and alter eicosanoid biosynthesis in platelets. ${ }^{1}$ They are commonly used as ingredients in various recipes or as herbs of therapeutic value in alternative medicine for treating inflammatory conditions. They are known causes of acquired platelet disorder but their role in triggering TTP is yet to be clarified. Herein, we report a case of acquired TTP primarily complicated by myocardial damage, which might have been triggered by Cumin and Turmeric consumption.

\section{Case presentation}

A previously healthy 46-year old African American man presented with itching, redness, swelling and tingling in palms after consuming turmeric and cumin as an ingredient of a new recipe. A presumptive diagnosis of an allergic reaction to these spices was made and he was treated with Prednisone, Hydroxyzine and Famotidine in the ED after which he was discharged on the same day. 2days later, the patient revisited the Emergency Department after noticing red colored urine, chest discomfort, sputum mixed with blood and dark black stools. Physical examination revealed conjunctival pallor and dark red stool on digital rectal examination. Vitals were unremarkable except for tachycardia of $127 \mathrm{bpm}$.

12 lead EKG showed $\mathrm{T}$ wave inversion in the lateral leads. Significant laboratory findings included hemoglobin of $10.7 \mathrm{~g} / \mathrm{dl}$, platelets of $13000 / \mathrm{mm} 3$, lactate dehydrogenase of $1499 \mathrm{IU} / \mathrm{L}$ and creatinine level of $1.9 \mathrm{mg} / \mathrm{dl}$. Further testing showed elevated D-dimer and Troponin I of $1.09 \mathrm{ng} / \mathrm{ml}$. Transaminases and disseminated intravascular coagulation panel were all normal. A peripheral blood film showed characteristic findings of microangiopathic hemolytic anemia (Figure 1).

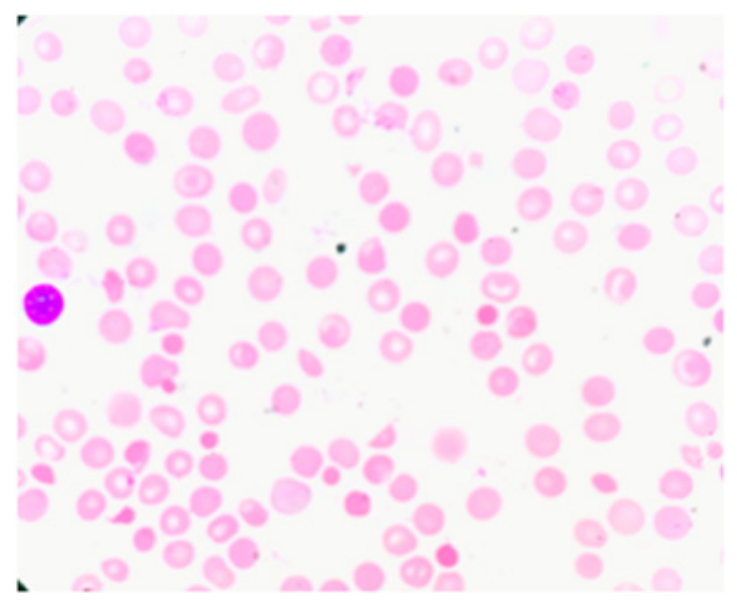

Figure I Fragmented RBCs (Schistocytes).

Emergent plasmapheresis (PEX) was initiated as soon as the clinical diagnosis was made. Later, ADAMTS13 activity came back less than $10 \%$ which confirmed the diagnosis of TTP. During the 
third cycle, PEX was complicated by 5 episodes of non-sustained Ventricular Tachycardia following which Amiodarone was rightly instituted. 2D Echocardiogram showed EF of 50\% and lateral wall hypokinesis. Troponin trend showed persistent elevations which suggested true cardiac muscle damage. Conventional management of myocardial infarction including cardiac catheterization was deferred because of severe thrombocytopenia and increased bleeding risk. Daily PEX continued until bleeding ceased, Troponin and platelet count normalised and patient recovered.

\section{Discussion}

Thrombotic thrombocytopenic purpura (TTP) is a fulminant disease characterized by a pentad of clinical features- fever, microangiopathic hemolytic anemia (MAHA), thrombocytopenia, neurologic and renal abnormalities. The annual incidence of Thrombotic Thrombocytopenic Purpura (TTP) currently has been estimated to be 11 per million populations ${ }^{2}$ compared to previous studies which reported incidence as 3.7-3.8 cases per million-person years. ${ }^{3,4}$ The incidence rate appears to be on the rise because of less stringent criteria and better resources used for diagnosis. It has been demonstrated that neurological features, renal failure, and fever were not present in all patients with TTP. ${ }^{5,6}$ Thus, the presence of microangiopathic hemolytic anemia and thrombocytopenia without an apparent cause is now considered sufficient to make a presumptive diagnosis of TTP.

TTP has 2 distinct clinical forms, congenital and acquired. In the congenital form, Upshaw-Schulman syndrome (USS), ADAMTS13 deficiency is due to mutations in gene encoding for ADAMTS $13^{7,8}$ whereas in acquired TTP it is due to the production of inactivating autoantibodies (esp IgG) against ADAMTS13. Infection (HIV), drugs (Ticlopidine, antineoplastic agents, immunosuppressive agents), malignancy, bone marrow transplantation and pregnancy may all be underlying etiological causes of acquired TTP, but the majority of cases are idiopathic. ${ }^{9}$

Cumin and Turmeric are commonly used spices in Asian foods. Also, they play an important role in alternative medicine for treating various inflammatory conditions. They are well known to cause platelet dysfunction by inhibiting arachidonate-induced platelet aggregation and eicosanoid synthesis. ${ }^{10}$ Their role in the pathogenesis of TTP is not yet well defined. As the evidence is strongly convincing, we hypothesize that severe deficiency of ADAMTS13 might have been triggered by the use of those spices in our case.

TTP most commonly involves the brain, kidneys but virtually any organ in the body can be affected, including the heart. As per the literature, the spectrum of cardiac involvement in TTP patients ranges from myocardial infarction, tachyarrhythmias, conduction disturbances, heart failure and cardiogenic shock to sudden cardiac death. ${ }^{11}$ The patient reported here had myocardial involvement early in course of the disease which was depicted by EKG changes, Troponin I elevation and Echocardiogram changes, all suggestive of acute ischemia. Acute Myocardial Infarction (AMI) is reported to be the most common cardiac manifestation of TTP. ${ }^{12-15}$ As per studies, the incidence of myocardial infarction as cardiac involvement was ranging from 15 to 40 percent in patients with TTP. ${ }^{12,15,16}$

Management of cardiac complications in acute episode of TTP becomes a challenge for the majority of physicians. The use of Aspirin (ASA) in thrombocytopenia is avoided as it increases the risk of bleeding, even though clinical trials depicted its role in prevention of platelet aggregation and reduction of mortality. ${ }^{17}$ Role of adding Clopidogrel into the treatment regimen is controversial as Clopidogrel itself can cause TTP. ${ }^{18}$ Due to severe thrombocytopenia and renal failure in TTP, cardiac catheterization cannot be performed. ${ }^{12}$ Hence, the cardiac complications cannot be managed by conventional therapy due to potential complications associated with it, but instead it can be treated by regular PEX, corticosteroids or Rituximab. Timely initiation of therapy reduces mortality and reverses ischemic cardiomyopathy as seen in our patient.

Plasma Exchange remains the therapy of choice, which has an $80 \%$ success rate and is often curative. ${ }^{19}$ The clinical improvement is a result of the reduction of circulating antibodies which inactivate ADAMTS13 and likely also removal of large vWF multimers. ${ }^{19}$ Patients diagnosed with severe deficiency of ADAMTS13, i.e. $<10 \%$, may also benefit by addition of Rituximab to PEX. ${ }^{20}$ In the era before effective treatment with plasma exchange, 90 percent of patients with TTP died from systemic microvascular thrombosis. With plasma exchange as primary treatment, mortality rate decreased to 10 to $20 \% .^{19,21}$

Survivors of acute TTP usually do not exhibit sequelae except a small percentage of patients with residual neurological deficits. The relapsing nature of this disease should not be overlooked. About 20$50 \%$ of patients exhibit relapse after an episode of idiopathic TTP, with the majority occurring in the first two years, with an estimated risk of $43 \%$ for relapse at 7.5 years. ${ }^{22,23}$ Factors attributed to increased risk of relapse are critically low levels of ADAMTS13 and high titers of autoantibodies against ADAMTS13 during an acute TTP episode. During remission, persistence of autoantibodies also increases the risk of relapse..$^{22,23}$ Relapse episodes are usually treated with corticosteroids, immunosuppressive therapy or Rituximab as a second line agent.

\section{Conclusion}

Even though Cumin and Turmeric induced TTP has never been reported before, this association cannot be ignored because of the evidence we provided above. Further evaluation in the future will be necessary to strengthen this link. Also, primary involvement of myocardium in acute TTP was shown to be significant so that serial EKGs, trending Cardiac Troponin levels and Telemetry monitoring during the initial assessment should become routine practice in patients with TTP.

\section{Acknowledgments}

We thank our program director, associate program director and attendings.

\section{Conflicts of interest}

Author declares there is no conflicts opf interest.

\section{Funding}

None.

\section{References}

1. Srivastava KC. Extracts from two frequently consumed spices--cumin (Cuminum cyminum) and turmeric (Curcuma longa)--inhibit platelet aggregation and alter eicosanoid biosynthesis in human blood platelets. Prostaglandins Leukot Essent Fatty Acids. 1989;37(1):57-64.

2. Terrell DR, Williams LA, Vesely SK, et al. The incidence of thrombotic thrombocytopenic purpura-hemolytic uremic syndrome: all patients, idiopathic patients, and patients with severe ADAMTS-13 deficiency. $J$ Thromb Haemost . 2005;3(7):1432-1436. 
3. Amorosi E, Ultmann J. Thrombotic thrombocytopenic purpura: report of 16 cases and review of the literature. Medicine. 1966;45(2):139-159.

4. Torok TJ, Holman RC, Chorba TL. Increasing mortality from thrombotic thrombocytopenic purpura in the United States-Analysis of national mortality data, 1968-1991. Am J Hematol. 1995;50(2):84-90.

5. Bell WR, Braine HG, Ness PM, et al. Improved survival in thrombotic thrombocytopenic purpura-hemolytic uremic syndrome. Clinical experience in 108 patients. Engl J Med. 1991;325(6):398-403.

6. Tsai HM, Lian ECN. Antibodies to von Willebrand factor-cleaving protease in acute thrombotic thrombocytopenic purpura. Engl J Med. 1998;339(22):1585-1594.

7. Kinoshita S, Yoshioka A, Park YD, et al. Upshaw-Schulman syndrome revisited: a concept of congenital thrombotic thrombocytopenic purpura. Int J Hematol. 2001;74(1):101-108.

8. Upshaw JD, Reidy TJ, Groshart K. Thrombotic thrombocytopenic purpura in pregnancy: response to plasma manipulations. South Med J. 1985;78(6):677-680.

9. Gordon LI, Kwaan HC. Thrombotic microangiopathy manifesting as thrombotic thrombocytopenic purpura/hemolytic uremic syndrome in the cancer patient. Semin Thromb Hemost. 1999;25(2):217-221.

10. Hawkins BM, Abu-Fadel M, Vesely SK, et al. Clinical cardiac involvement in thrombotic thrombocytopenic purpura: a systematic review. Transfusion. 2008;48(2):382-392.

11. Wahla AS, Ruiz J, Noureddine N, et al. Myocardial infarction in thrombotic thrombocytopenic purpura: a single-center experience and literature review. Eur J Haematol. 2008; 81(4):311-316.

12. Podolsky SH, Zembowicz A, Schoen FJ, et al. Massive myocardial necrosis in thrombotic thrombocytopenic purpura: a case report and review of the literature. Arch Pathol Lab Med. 1999;123(10):937-940.

13. Perez L, Ramappa P, Guzman JA. Myocardial injury in thrombotic thrombocytopenic purpura: a frequent, perplexing complication. Int $J$ Cardiol. 2008;128(2):257-260.
14. Patschan D, Witzke O, Duhrsen U, et al. Acute myocardial “ infarction in thrombotic microangiopathies - clinical characteristics, risk factors and outcome. Nephrol Dial Transplant. 2006;21(6):1549-1554.

15. McCarthy LJ, Danielson CF, Skipworth EM, et al. Myocardial infarction/ injury is relatively common at presentation of acute thrombotic thrombocytopenic purpura: the Indiana University experience. Ther Apher. 2002;6(1):2-4.

16. Gaddam S, Pablani L, Chainani V, et al. Complete recovery of ischemic cardiomyopathy from thrombotic thrombocytopenic purpura. Clin Med Insights Cardiol . 2011;5:29-33.

17. Bennett CL, Connors JM, Carwile JM, et al. Thrombotic thrombocytopenic purpura associated with clopidogrel. $N$ Engl J Med 2000;342(24):1773-1777.

18. George JN. Thrombotic thrombocytopenic purpura. $N$ Engl J Med 2006;354(18):1927-1935

19. George JN. How I treat patients with thrombotic thrombocytopenic purpura: 2010. Blood. 2010;116:4060-4069.

20. Kessler CS, Khan BA, Lai-Miller K. Thrombotic thrombocytopenic purpura: a hematological emergency. JEmerg Med. 2012;43(3):538-544.

21. George JN, Al-Nouri ZL. Diagnostic and therapeutic challenges in the thrombotic thrombocytopenic purpura and hemolytic uremic syndromes. Hematology Am Soc Hematol Educ Program. 2012;2012:604-609.

22. Chaturved S, Carcioppolo D, Zhang L, et al. Management and outcomes of patients with TTP: analysis of 100 cases at a single institution. $A m ~ J$ Hematol .2013;88(7):560-565.

23. Heesun J Rogers, Charles Allen, Alan E Lichtin. Thrombotic thrombocytopenic purpura: The role of ADAMTS13. Cleve Clin J Med. 2016;83(8):597-603. 